University of New Orleans

ScholarWorks@UNO

7-1975

\title{
Combined reflection and transmission thin-film ellipsometry: a unified linear analysis
}

Rasheed M.A. Azzam

University of New Orleans, razzam@uno.edu

M. Elshazly-Zaghloul

N. M. Bashara

Follow this and additional works at: https://scholarworks.uno.edu/ee_facpubs

Part of the Electrical and Electronics Commons

\section{Recommended Citation}

R. M. A. Azzam, M. Elshazly-Zaghloul, and N. M. Bashara, "Combined reflection and transmission thin-film ellipsometry: a unified linear analysis," Appl. Opt. 14, 1652-1663 (1975) http://www.opticsinfobase.org/ ao/abstract.cfm?URI=ao-14-7-1652

This Article is brought to you for free and open access by the Department of Electrical Engineering at ScholarWorks@UNO. It has been accepted for inclusion in Electrical Engineering Faculty Publications by an authorized administrator of ScholarWorks@UNO. For more information, please contact scholarworks@uno.edu. 


\title{
Combined reflection and transmission thin-film ellipsometry: a unified linear analysis
}

\author{
R. M. A. Azzam, M. Elshazly-Zaghloul, and N. M. Bashara
}

\begin{abstract}
A scheme of combined reflection and transmission ellipsometry on light-transmitting ambient-film-substrate systems is proposed and the required sample design and instrument operation are investigated. A comparative study of the sensitivity of external and internal reflection and transmission ellipsometry is carried out based on unified linear approximations of the exact equations. These approximations are general in that an arbitrary initial film thickness is assumed. They are simple, because a complex sensitivity function is introduced whose real and imaginary projections determine the psi $(\psi)$ and delta $(\Delta)$ sensitivity factors. Among the conclusions of this paper are the following. (1) External reflection ellipsometry near the Brewster angle of a transparent ambient-substrate system is extremely sensitive to the presence of very thin interfacial films. For example, films as thin as $10^{-5} \AA$ of gold are readily detectable on glass substrates at an angle of incidence $0.3^{\circ}$ below the Brewster angle, assuming a measuring wavelength of $5461 \AA$ with an ellipsometer of $0.05^{\circ}$ precision. (2) The formation of thin nonabsorbing films at the interface between transparent ambient and substrate media is not detectable, to first order, as a change in the ellipsometric angle $\psi$ by either internal or external reflection or transmission ellipsometry. (3) The film-detection sensitivity of transmission ellipsometry increases monotonically with angle of incidence. (4) For each angle of external incidence there is a corresponding angle of internal incidence that leads to the same values of the reflection and transmission sensitivity functions. These angles are interrelated by Snell's law. (5) The ranges of validity of the linear approximation in reflection and transmission ellipsometry are comparable. The case of total internal reflection ellipsometry may lead to strong nonlinear behavior of $\psi$ and $\Delta$ as functions of the film thickness in the range below 0.05 of the wavelength of light.
\end{abstract}

\section{Introduction}

The formation of a thin-film phase at the interface between an ambient and a substrate can be conveniently monitored, in an essentially nondestructive manner, by a light-beam probe. Several techniques have employed this principle, such as ellipsometry ${ }^{1}$ and absolute and differential reflectance spectroscopy. ${ }^{2,3}$ From these techniques, we can obtain the effective optical thickness $d_{f}$ and the effective complex refractive index $N_{f}=n_{f}-j k_{f}$ of the thin-film phase. $n_{f}$ and $k_{f}$ are the real refractive index and extinction coefficient of the film, respectively.

Ellipsometry is usually carried out on the reflected wave at a given angle of incidence. The optical properties of the ambient and substrate media are generally known or can be easily measured. The quantity measured by the ellipsometer is the ratio $R_{p} / R_{s}=$ $\tan \psi \exp (j \Delta)$ of the complex amplitude-reflection

The authors are with the Electrical Materials Laboratory, College of Engineering, University of Nebraska, Lincoln, Nebraska 68508

Received 20 August 1974. coefficients $R_{p}$ and $R_{s}$ for light polarized with its electric vector vibrating parallel $(p)$ and perpendicular (s) to the plane of incidence, respectively. Measurements of the ellipsometric parameters $\psi$, the angle whose tangent is the relative amplitude attenuation of the $p$ and $s$ vibrations, and $\Delta$, the difference between the phase shifts of these two vibrations induced by reflection, are often adequate for the determination of the thickness $d_{f}$ and real refractive index $n_{f}$ of a nonabsorbing film. However, when the film is absorbing, an additional measurement, for example, of the reflectance $\mathbf{R}$, transmittance $\mathbf{T}$, or $\psi$ and $\Delta$ at another angle of incidence, is required to determine or overdetermine the three parameters $d_{f}, n_{f}$, and $k_{f}$ of the film. ${ }^{1,2}$

An interesting possibility that has received little or no attention is that of combined ellipsometry on the reflected and transmitted waves at a single angle of incidence to determine $d_{f}, n_{f}$, and $k_{f}$. In this case, the ambient and substrate media should be transparent and the film must be thin enough for the transmitted wave to have measurable intensity. ${ }^{4}$ Also, if light is incident on the interface from the bulk phase of higher refractive index, the angle of incidence must be chosen below the critical angle of total inter- 


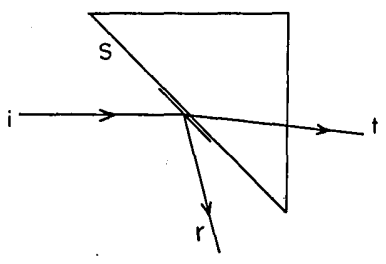

a

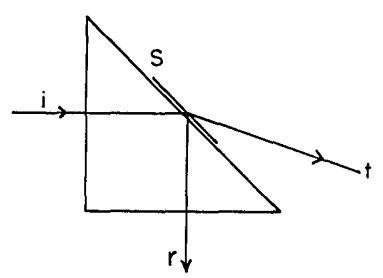

b

Fig. 1. Prism substrate for fixed-angle-of-incidence operation; (a) external incidence and (b) internal incidence.

nal reflection; otherwise, the transmitted wave becomes evanescent, hence inaccessible for measurement.

It is the purpose of this paper to investigate this method of combined reflection and transmission ellipsometry on three-phase systems with particular emphasis on a comparative study of the sensitivity of the ellipsometric parameters $\psi_{R}, \Delta_{R}$ and $\psi_{T}, \Delta_{T}$ that characterize light reflection and transmission at the interface, respectively, to the growth of an intermediate thin-film phase. Such sensitivity considerations will be based on new linear approximations of the exact equations of reflection and transmission ellipsometry that are both simple and general; the range of validity of these approximations will also be considered. The case of vacuum deposition of dielectric, semiconducting, and metallic films on glass substrates is taken as an example.

\section{Substrate-Element Design and Instrument Set-up}

The transparent substrate may be shaped either as a prism or semicylinder, dependent on whether fixed or variable-angle-of-incidence operation is desired, respectively. Figure 1 shows a prism substrate suitable for fixed-angle-of-incidence operation. Light is incident either externally (a) or internally (b) at an oblique angle on one planer face of the prism in contact with the surrounding ambient where film formation is to take place. The light beam must enter or leave the substrate medium at normal incidence to the other prism faces. Figure 2 shows a semicylindrical substrate that can be rotated around its axis to vary the angle of incidence. Light enters or leaves the substrate at normal incidence to its cylindrical surface. The active surface in this case is the planar face of the semicylinder that is in contact with the ambient. Light strikes this face obliquely and may come from the ambient side (external reflection, case a) or from the substrate side of the interface (internal incidence, case b). The important requirements in the design of the substrate element are (1) that it be transparent, (2) that it exhibit the least possible residual stress birefringence, and (3) that it have such geometry that light enters or leaves at normal incidence to the nonactive surfaces that may be antireflection-coated. If the ambient is not the atmosphere of the test site itself, the substrate element has to be enclosed in a cell with entrance and exit optical windows. In this case, the nonactive surfaces of the substrate element can serve as entrance or exit faces of the cell, thereby minimizing total instrument birefringence.

Combined ellipsometry on the reflected and transmitted waves may require no modification of existing instruments. Thus, the analyzer telescope (arm) of a conventional ellipsometer first can be set to measure the reflected beam, then is rotated to measure the transmitted beam. From such measurements we obtain $\left(\psi_{R}, \Delta_{R}\right)$ and $\left(\psi_{T}, \Delta_{T}\right)$ that characterize light reflection and transmission at the ambient-substrate interface, respectively. The instrument can be operated as a null ellipsometer, rotating-analyzer ellipsometer $^{5-7}$ (RAE), or as polarization-modulated ellipsometer $^{8}$ (PME).

When a rapid surface reaction is to be followed, rotation of the analyzer telescope of a conventional ellipsometer between the reflected and transmitted beams may become unacceptably slow. Under such conditions, it may become necessary to equip the instrument with a second independent analyzer telescope that can also be rotated around the central axis of the ellipsometer (Fig. 3). During a given experiment, both telescopes remain fixed and the reflected and transmitted beams are sensed simultaneously and independently.

When this three-telescope instrument is operated in the null mode, it is preferable to keep the elements

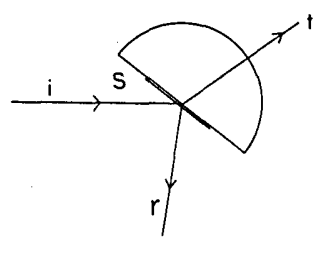

a

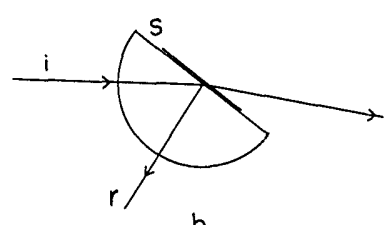

b

Fig. 2. Semicylindrical substrate for variable-angle-of-incidence operation; (a) external incidence and (b) internal incidence. 


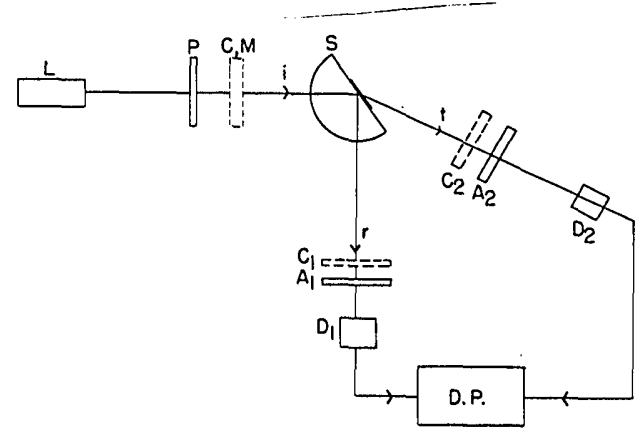

Fig. 3. Three-telescope ellipsometer for combined reflection and transmission ellipsometry. $L$ is the light source and D.P. is the data processor. The other symbols are explained in the text.

in the incident-beam telescope stationary. This can be achieved by use of a polarizer $P$ in the incidentbeam telescope and fitting each of the reflected-beam and transmitted-beam telescopes with a compensator $C$ and analyzer $A$ (Fig. 3). The polarizer $P$ is set at a suitable fixed azimuth from the plane of incidence, while the compensator $C_{1}$ and analyzer $A_{1}$ are servoed to null the reflected beam ${ }^{9}$ (output of $D_{1}$ ) and the compensator $C_{2}$ and the analyzer $A_{2}$ are servoed to null the transmitted beam (output of $D_{2}$ ). These nulls must be dynamically maintained as a surface reaction evolves. From the angular null settings $C_{1 n}$, $A_{1 n}$, we obtain $\psi_{R}, \Delta_{R}$ and from $C_{2 n}, A_{2 n}$ we obtain $\psi_{T}, \Delta_{T}$.

The above null ellipsometer is uneconomical in its use of two compensators and four servomotors to drive $C_{1}, A_{1}, C_{2}$, and $A_{2}$. The speed of mechanical servonulling is also limited and convergence to the null by the compensator and analyzer is a multistep, slow process. ${ }^{10}$ For these reasons, RAE or PME ellipsometers are better candidates for the present application when compared to null ellipsometers. In RAE, the compensators $C_{1}$ and $C_{2}$ are removed, a compensator $C$ may (or may not) be placed in the incident-beam telescope after the polarizer, and the analyzers $A_{1}$ and $A_{2}$ are synchronously rotated around their respective beams. In PME, compensators $C_{1}$ and $C_{2}$ are also removed, a fixed piezomodulator $M$ driven by a high frequency oscillator is inserted in the incident-beam telescope after the polarizer, and the analyzers $A_{1}$ and $A_{2}$ are set in their respective beams at suitable fixed azimuths. The advantages of using RAE or PME lie in the feasibility of very rapid sequential sampling of the outputs of the two detectors $D_{1}$ and $D_{2}$, which can be Fourier-analyzed to yield $\psi_{R}, \Delta_{R}$ and $\psi_{T}, \Delta_{T}$, respectively. On-line data reduction is possible by a minicomputer ${ }^{6-8}$ with input data supplied by both the reflected-beam and transmitted-beam information channels.

\section{Linear Approximations of the Equations of Reflection and Transmission Ellipsometry}

\section{A. Reflection}

In reflection ellipsometry on an optically isotropic three-phase system (bulk phase 0 , film phase 1 , bulk phase 2 ), we determine the ratio ${ }^{1,2}$

$$
\rho=R_{p} / R_{s}
$$

of the overall complex amplitude-reflection coefficients

$$
R_{\nu}=\left[r_{01 \nu}+r_{12 \nu} \exp (-j 2 \beta)\right] /\left[1+r_{01 \nu} r_{12 \nu} \exp (-j 2 \beta)\right],
$$

for light polarized parallel $(\nu=p)$ and perpendicular $(\nu=s)$ to the plane of incidence. $r_{01 \nu}, r_{12 \nu}(\nu=p, s)$ represent the 0-1 and 1-2 interface Fresnel reflection coefficients for the $p$ and $s$ polarizations and

$$
\beta=2 \pi\left(d_{1} / \lambda\right)\left(N_{1}^{2}-N_{0}^{2} \sin ^{2} \phi_{0}\right)^{1 / 2}
$$

is the change in the temporal phase of the wave caused by its traversal of the film (phase 1) whose thickness is $d_{1}$. Lambda is the free-space wavelength of light. $N_{0}$ is the refractive index of phase 0 in which light is incident on the $0-1$ interface at an angle $\phi_{0}$.

The main equation of reflection ellipsometry, which results from combining Eqs. (1) and (2), is rather complicated. When the thickness of the film phase changes by a small increment in comparison with the wavelength of light, a linear approximation can be used with reasonable accuracy. Such approximation, valid for any optically isotropic ambientfilm-substrate system with an arbitrary initial film thickness, will now be derived.

By taking the logarithmic differential of Eq. (1), we obtain

$$
\delta \rho / \rho=\left(\delta R_{p} / R_{p}\right)-\left(\delta R_{s} / R_{s}\right),
$$

which states that the fractional change $(\delta \rho / \rho)$ of the ellipsometric quantity $\rho$ is equal to the difference between the fractional changes $\left(\delta R_{p} / R_{p}\right)$ and $\left(\delta R_{s} / R_{s}\right)$ of the complex amplitude-reflection coefficients $R_{p}$ and $R_{s}$ for the $p$ and $s$ polarizations, respectively. To determine the latter fractional changes, we write Eq. (2) as

where

$$
R_{\nu}=N_{\nu} / D_{\nu}
$$

and

$$
N_{\nu}=r_{01 \nu}+r_{12 \nu} \xi, \quad D_{\nu}=1+r_{01 \nu} r_{12 \nu} \xi,
$$

$$
\begin{gathered}
\xi=\exp (-j 2 \beta)=\exp \left(\gamma d_{1}\right), \\
\gamma=-j(4 \pi / \lambda)\left(N_{1}^{2}-N_{0}^{2} \sin ^{2} \phi_{0}\right)^{1 / 2} .
\end{gathered}
$$

The logarithmic differential of Eq. (5) is

$$
\delta R_{\nu} / R_{\nu}=\left(\delta N_{\nu} / N_{\nu}\right)-\left(\delta D_{\nu} / D_{\nu}\right) .
$$

Above and throughout, all equations with $\nu$ subscripts should be considered applicable for both $\nu=p$ and $\nu=s$. When all differential changes are induced by an incremental change $\delta \xi$ of the complex exponential function $\xi$, Eq. (7), we can rewrite Eq. (9) as

$$
\delta R_{\nu} / R_{\nu}=\left[\left(1 / N_{\nu}\right)\left(d N_{\nu} / d \xi\right)-\left(1 / D_{\nu}\right)\left(d D_{\nu} / d \xi\right)\right] \delta \xi .
$$

If, in turn, the increment $\delta \xi$ of $\xi$ results from an increment $\delta d_{1}$ of the film thickness $d_{1}$, from Eq. (7) we get

$$
\delta \xi=\gamma \dot{\xi} \delta d_{1} .
$$


Substituting Eq. (11) and Eqs. (6) and their derivatives

$$
d N_{\nu} / d \xi=r_{12 \nu}, \quad d D_{\nu} / d \xi=r_{01 \nu} r_{12 \nu}
$$

into Eq. (10), we find that

$$
\begin{aligned}
\delta R_{\nu} / R_{\nu}= & \gamma d_{1} \xi\left\lfloor\mid r_{12 \nu} /\left(r_{01 \nu}+r_{12 \nu} \xi\right)\right] \\
& \left.-\left[r_{01 \nu} r_{12 \nu} /\left(1+r_{01} r_{12 \nu} \xi\right)\right]\right\}\left(\delta d_{1} / d_{1}\right) \\
= & Q_{\nu}\left(\delta d_{1} / d_{1}\right),
\end{aligned}
$$

where

$$
\begin{aligned}
Q_{\nu}=\gamma d_{1} \xi\left\{\left[r_{12 \nu} /\left(r_{01 \nu}+\right.\right.\right. & \left.\left.r_{12 \nu} \xi\right)\right] \\
& \left.-\left[r_{01 \nu} r_{12 \nu} /\left(1+r_{01 \nu} r_{12 \nu} \xi\right)\right]\right\} .
\end{aligned}
$$

Equations (13) and (14) are important because they relate a fractional change of film thickness $\left(\delta d_{1} /\right.$ $d_{1}$ ) to the resulting fractional change of the overall (three-phase) complex amplitude-reflection coefficient $^{11} \cdot\left(\delta R_{\nu} / R_{\nu}\right)$ for the two fundamental polarizations $\nu=p$ and $\nu=s$ through complex coupling coefficients $Q_{\nu}$.

In ellipsometry, we are interested in the fractional change $\delta \rho / \rho$ of the ratio of reflection coefficients $\rho$ that is caused by a fractional change $\delta d_{1} / d_{1}$ of film thickness $d_{1}$. This is obtained by substituting from Eqs. (13) and (14) into Eq. (4)

$$
\begin{aligned}
& \qquad \delta \rho / \rho=K\left(\delta d_{1} / d_{1}\right), \\
& \text { where } \quad K=Q_{p}-Q_{s}, \\
& =\gamma d_{1} \xi\left\{\left[r_{12 p} /\left(r_{01 p}+r_{12 p} \xi\right)\right]\right. \\
& -\left[r_{01 p} r_{12 p} /\left(1+r_{01 p} r_{12 p} \xi\right)\right]-\left[r_{12 s} /\left(r_{01 s}+r_{12 s} \xi\right)\right] \\
& \left.+\left[r_{01 s} r_{12 s} /\left(1+r_{01 s} r_{12 s} \xi\right)\right]\right\} .
\end{aligned}
$$

The quantity $K$, Eq. (16), is significant because it defines a complex sensitivity factor that couples a fractional change of film thickness $\delta d_{1} / d_{1}$ to a corresponding fractional change of the ellipsometric ratio of reflection coefficients $\delta \rho / \rho$, Eq. (15).

The above results are general in that the small changes of film thickness $\delta d_{1}$ are assumed to take place in the neighborhood of an arbitrary initial value $d_{1}$. Many cases of practical interest involve the growth of very thin films at the interface between an ambient and substrate starting from zero film thickness $d_{1}$. In this case, we desire to relate $\delta R_{\nu} / R_{\nu}$ and $\delta \rho / \rho$ directly to $\delta d_{1}$, instead of $\delta d_{1} / d_{1}$, which becomes infinite when $d_{1}=0$. The equations that apply when the initial film thickness is zero can be readily obtained by setting $\xi=1$ [from Eq. (7), $\xi=1$ when $d_{1}=$ 0] in Eqs. (13)-(16). They are listed below

$$
\begin{gathered}
\delta R_{\nu} / R_{\nu}=\gamma\left[\left\{r_{12 \nu} /\left(r_{01 \nu}+r_{12 \nu}\right)\right]\right. \\
\left.\quad-\left[r_{01 \nu} r_{12 \nu} /\left(1+r_{01 \nu} r_{12 \nu}\right)\right]\right\} \delta d_{1} \\
=\bar{Q}_{\nu} \delta d_{1}, \\
\bar{Q}_{\nu}=\gamma\left\{\left[r_{12 \nu} /\left(r_{01 \nu}+r_{12 \nu}\right)\right]\right. \\
\left.-\left[r_{01 \nu} r_{12 \nu} /\left(1+r_{01 \nu} r_{12 \nu}\right)\right]\right\} \\
\delta \rho / \rho=\bar{K} \delta d_{1}, \\
\bar{K}=\bar{Q}_{p}-\bar{Q}_{s},
\end{gathered}
$$

$$
\begin{aligned}
= & \gamma\left\{\left[r_{12 p} /\left(r_{01 p}+r_{12 p}\right)\right]-\left[r_{01 p} r_{12 p} /\left(1+r_{01 p} r_{12 p}\right)\right]\right. \\
& \left.-\left[r_{12 s} /\left(r_{01 s}+r_{12 s}\right)\right]+\left[r_{01 s} r_{12 s} /\left(1+r_{01 s} r_{12 s}\right)\right]\right\} .
\end{aligned}
$$

Recall that $\gamma$ appearing in the above equations is given by Eq. (8).

\section{B. Transmission}

In transmission ellipsometry, we determine the ratio

$$
\rho=T_{p} / T_{s},
$$

of the overall complex-amplitude transmission coefficients

$$
T_{\nu}=t_{01 \nu} t_{12 \nu} \exp (-j \beta) /\left[1+\gamma_{01 \nu} r_{12 \nu} \exp (-j 2 \beta)\right],
$$

for light polarized parallel $(\nu=p)$ and perpendicular $(\nu=s)$ to the plane of incidence. $t_{01 \nu}, t_{12 \nu}(\nu=p, s)$ represent the $0-1$ and 1-2 interface transmission coefficients for the $p$ and $s$ polarizations; the other quantities are the same as defined earlier for reflection ellipsometry. The main equation of transmission ellipsometry can be obtained by combining Eqs. (21) and (22)

$$
\begin{aligned}
\rho & =\left(t_{01 p} / t_{01 s}\right)\left(t_{12 p} / t_{12 s}\right)\left\{\left[1+\gamma_{01 s} r_{12 s} \exp (-j 2 \beta)\right] /\right. \\
& {\left.\left[1+r_{01 p} r_{12 p} \exp (-j 2 \beta)\right]\right\} } \\
& =M\left(D_{s} / D_{p}\right),
\end{aligned}
$$

where

$$
M=\left(t_{01 p} / t_{01 s}\right)\left(t_{12 p} / t_{12 s}\right),
$$

and $D_{\nu}(\nu=p, s)$ are the same as in Eqs. (6). By taking the logarithmic differential of Eq. (23), we obtain

$$
\delta \rho / \rho=(\delta M / M)+\left(\delta D_{s} / D_{s}\right)-\left(\delta D_{p} / D_{p}\right) .
$$

If all differential changes arise from changes of film thickness alone, $\delta M / M=0$, and Eq. (25) simplifies to

$$
\begin{aligned}
\delta \rho / \rho= & \left(\delta D_{s} / D_{s}\right)-\left(\delta D_{p} / D_{p}\right) \\
& =\left[\left(1 / D_{s}\right)\left(d D_{s} / d \xi\right)-\left(1 / D_{p}\right)\left(d D_{p} / d \xi\right)\right] \delta \xi .
\end{aligned}
$$

$\delta \xi$ is the differential change of the complex exponential function $\xi$, Eq. (7), that corresponds to a change of film thickness $\delta d_{1}$. If we substitute $D_{\nu}$ and their derivatives $d D_{\nu} / d \xi(\nu=p, s)$ from Eqs. (6) and (12), respectively, and $\delta \xi$ from Eq. (11) into Eq. (26), we get

$$
\begin{aligned}
\delta \rho / \rho & =-\gamma d_{1} \xi\left\{\left[r_{01 p} \gamma_{12 p} /\left(1+\gamma_{01 p} r_{12 p} \xi\right)\right]\right. \\
& \left.-\left[r_{01 s} \gamma_{12 s} /\left(1+r_{01 s} r_{12 s} \xi\right)\right]\right\}\left(\delta d_{1} / d_{1}\right), \\
& =K\left(\delta d_{1} / d_{1}\right),
\end{aligned}
$$

where

$$
\begin{aligned}
K=-\gamma d_{1} \xi\left\{\left[\gamma_{01 p} r_{12 p} /(1\right.\right. & \left.\left.+r_{01 p} r_{12 p} \xi\right)\right] \\
- & {\left.\left[r_{01 s} r_{12 s} /\left(1+\gamma_{01 s} \gamma_{12 s} \xi\right)\right]\right\} . }
\end{aligned}
$$

Equation (27) shows that $K$ of Eq. (28) defines a complex sensitivity factor that couples a fractional change of film thickness $\delta d_{1} / d_{1}$ to a corresponding fractional change $\delta \rho / \rho$ of the ellipsometric ratio of transmission coefficients. Equations (27) and (28) 
apply when film-thickness changes $\delta d_{1}$ occur around an arbitrary initial value $d_{1}$. For film growth starting from zero thickness, we set $\xi=1$ in Eq. (27)

$$
\begin{aligned}
\delta \rho / \rho & =-\gamma\left\{\left[r_{01 p} r_{12 p} /\left(1+r_{01 \rho} r_{12 p}\right)\right]\right. \\
& \left.=\bar{K} \delta d_{1}, \quad-\left[r_{01 s} r_{12 s} /\left(1+r_{01 s} r_{12 s}\right)\right]\right\} \delta d_{1},
\end{aligned}
$$

where

$$
\begin{aligned}
\bar{K}=-\gamma\left\{\left[r_{01 p} r_{12 p} /\left(1+r_{01 p} r_{12 p}\right)\right]\right. & \\
& \left.-\left[r_{01 s} r_{12 s} /\left(1+r_{01 s} r_{12 s}\right)\right]\right\},
\end{aligned}
$$

and $\gamma$ is given by Eq. (8).

\section{C. $\psi$ and $\Delta$ Sensitivity Factors}

Instead of the fractional change $\delta \rho / \rho$ of the ratio of reflection or transmission coefficients, it is usually more convenient to deal with the corresponding changes $\delta \psi$ and $\delta \Delta$ of the ellipsometric angles $\psi$ and $\Delta$. By taking the logarithmic differential of

$$
\rho=\tan \psi \exp (j \Delta)
$$

we obtain

$$
\delta \rho / \rho=(2 \delta \psi / \sin 2 \psi)+j \delta \Delta,
$$

so that

$$
\begin{gathered}
\delta \psi=(1 / 2 \sin 2 \psi) \operatorname{Re}(\delta \rho / \rho), \\
\delta \Delta=\operatorname{Im}(\delta \rho / \rho) .
\end{gathered}
$$

$\operatorname{Re}(X)$ and $\operatorname{Im}(X)$ denote the real and imaginary parts of $X$. When $\delta \rho / \rho$ is caused by a fractional change of film thickness $\delta d_{1} / d_{1}$, we can rewrite Eqs. (33) as

$$
\begin{gathered}
\delta \psi=[(1 / 2 \sin 2 \psi) \operatorname{Re}(K)]\left(\delta d_{1} / d_{1}\right), \\
\delta \Delta=[\operatorname{Im}(K)]\left(\delta d_{1} / d_{1}\right),
\end{gathered}
$$

for an arbitrary initial film thickness $d_{1}$, or as

$$
\begin{gathered}
\delta \psi=[(1 / 2 \sin 2 \bar{\psi}) \operatorname{Re}(\bar{K})] \delta d_{1}, \\
\delta \Delta=[\operatorname{Im}(\bar{K})] \delta d_{1},
\end{gathered}
$$

for zero initial film thickness. Equations (34) and (35) are obtained by substituting $\delta \rho / \rho$ from Eq. (15) [or Eq. (19)] and Eq. (27) [or Eq. (29)] into Eqs. (33), respectively. Cast somewhat differently, Eqs. (34) and (35) read

$$
\begin{aligned}
\psi & =\psi_{0}+S_{\psi}\left(\delta d_{1} / d_{1}\right), \\
\Delta & =\Delta_{0}+S_{\Delta}\left(\delta d_{1} / d_{1}\right),
\end{aligned}
$$

and

$$
\begin{aligned}
\psi & =\bar{\psi}+\bar{S}_{\psi} \delta d_{1}, \\
\Delta & =\bar{\Delta}+\bar{S}_{\Delta} \delta d_{1},
\end{aligned}
$$

where

$$
\begin{gathered}
S_{\downarrow}=\left(1 / 2 \sin 2 \psi_{0}\right) \operatorname{Re}(K), \\
S_{\Delta}=\operatorname{Im}(K), \\
\bar{S}_{\downarrow}=(1 / 2 \sin 2 \bar{\psi}) \operatorname{Re}(\bar{K}), \\
\bar{S}_{\Delta}=\operatorname{Im}(\bar{K}) .
\end{gathered}
$$

$\psi_{0}, \Delta_{0}$ are the ellipsometric angles that correspond to an arbitrary initial film thickness $d_{1}$ and $\bar{\psi}, \bar{\Delta}$ are the same quantities when $d_{1}=0$ (i.e., the parameters characteristic of the film-free ambient-substrate interface). Equations (31)-(39) are the same for both reflection and transmission ellipsometry. $\bar{S}_{\psi R}, \bar{S}_{\psi T}$ and $\bar{S}_{\Delta R}, \bar{S}_{\Delta T}$ are psi and delta sensitivity factors ${ }^{12}$ that determine the extent to which small changes of thickness of the film phase influence the ellipse of polarization of the reflected and transmitted light beams. These quantities are therefore of prime importance and their magnitudes and behavior with angle of incidence are of great interest. If the complex sensitivity factors $K$ and $\bar{K}$ are expressed in terms of their magnitudes and angles

$$
K=|K| \exp (j \theta), \quad \bar{K}=|\bar{K}| \exp (j \bar{\theta}),
$$

the psi and delta sensitivity factors of Eqs. (38) and (39) become

and

$$
\begin{gathered}
S_{\downarrow}=(180 / \pi)\left(1 / 2 \sin 2 \psi_{0}\right)|K| \cos \theta, \\
S_{\Delta}=(180 / \pi)|K| \sin \theta,
\end{gathered}
$$

$$
\begin{gathered}
\bar{S}_{\psi}=(180 / \pi)(1 / 2 \sin 2 \bar{\psi})|\bar{K}| \cos \bar{\theta}, \\
\bar{S}_{\Delta}=(180 / \pi)|\bar{K}| \sin \bar{\theta},
\end{gathered}
$$

respectively, in units of degrees per angstrom. For reflection ellipsometry, the complex sensitivity factors $|K| \exp (j \theta),|\bar{K}| \exp (j \bar{\theta})$ that appear in Eqs. (41) and (42) are given by Eqs. (16) and (20), whereas for transmission ellipsometry, they are given by Eqs. (28) and (30).

The analysis presented in this section provides a unified treatment of the theory of linear approximation for reflection and transmission ellipsometry. The linear approximation of the equation of reflection ellipsometry differs from previous work ${ }^{13-20}$ in that an arbitrary initial nonzero film thickness is assumed. It is less complicated, because it starts with the fractional change of the ellipsometric ratio $\delta \rho / \rho$ and relates it to the fractional change of film thickness $\delta d_{1} / d_{1}$ through complex sensitivity factors $K$ and $\bar{K}$. By leaving the complex sensitivity factors $K$ and $\bar{K}$ expressed in terms of the interface Fresnel coefficients [Eqs. (16), (20), (28), and (30)], rather than the optical properties of the three media, considerable complexity is avoided and with it the possibility of erroneous results. ${ }^{21}$ Furthermore, we have seen that the psi and delta sensitivity factors $S_{\psi}$ and $S_{\Delta}$ are proportional to the projections $(|K| \cos \theta,|K| \sin \theta)$ of the complex sensitivity factor $K$ on the real and imaginary axes respectively, Eqs. (41) and (42).

In the following section, we apply the results of this theory to a comparative study of the sensitivity of reflection and transmission ellipsometry, taking as an example the vacuum deposition of $\mathrm{MgF}_{2}, \mathrm{Si}$, and $\mathrm{Au}$ films (which cover the range from nonabsorbing to highly absorbing materials) on glass substrates. The bulk optical constants of these materials will be assumed even when the film thickness is in the angstrom and subangstrom range. 


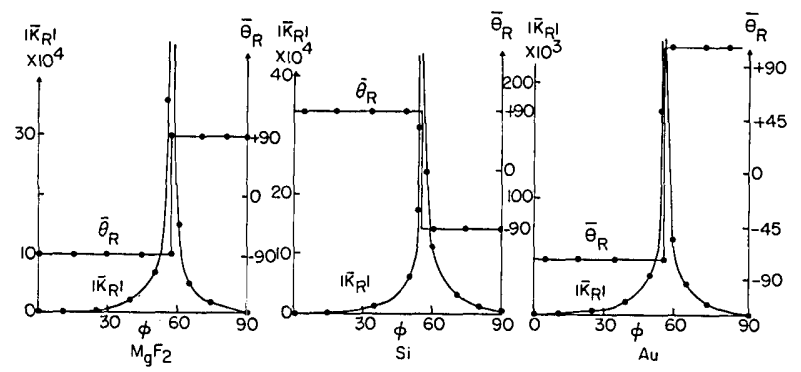

Fig. 4. Dependence of the magnitude $\left|\bar{K}_{R}\right|$ and angle $\bar{\theta}_{R}$ of the complex sensitivity function $\bar{K}_{R}$ on the angle of incidence $\phi$ for external reflection ellipsometry. In this and the subsequent figures $|\bar{K}|$ is in reciprocal angstroms and $\bar{\theta}$ and $\phi$ are in degrees. Left: air-MgF $\mathrm{M}_{2}$-glass; middle: air-Si-glass; right: air-Au-glass.

\section{Applications}

We have computed the complex sensitivity factor $\bar{K}[=|\bar{K}| \exp (j \bar{\theta})]$, and the real psi and delta sensitivity factors $\bar{S}_{\psi}$ and $\bar{S}_{\Delta}$ for the reflected and transmitted waves as functions of angle of incidence $\phi$ in case of film formation at an air (vacuum)-glass interface. The results obtained should be particularly useful, for example, to those interested in following the early stages of film growth on glass substrates $(N=1.5)$ by deposition of material from a vapor stream under vacuum $(N=1)$. It is assumed that the combined reflection and transmission ellipsometry technique proposed in this paper will be used. As a representative sample of film materials, we have chosen a dielectric [magnesium fluoride $\left(\mathrm{MgF}_{2}\right), N=1.38$ ], a semiconductor [silicon ( $\mathrm{Si}), N=4.05-j 0.028$ ], and a metal [gold (Au), $N=0.35-j 2.45$ ]. The wavelength of the ellipsometer light beam used is that of the mercury green line at $5461 \AA$. The refractive indices $N$ quoted above correspond to this wavelength. Both cases of external and internal incidence will be considered.

\section{A. External Incidence}

Light is incident from air(vacuum) on a film-covered glass substrate. Both the reflected and transmitted waves are monitored.

\section{Reflection Ellipsometry}

The magnitude $\rfloor \bar{K}_{R} \mid$ and angle $\bar{\theta}_{R}$ of the complex sensitivity factor $K_{R}$ are plotted vs the angle of inci- dence $\phi$ in Fig. 4 for the $\mathrm{MgF}_{2}, \mathrm{Si}$, and Au films. The most important features of these curves are the following.

(a) The magnitude $\left|\bar{K}_{R}\right|$ of the complex sensitivity factor $\bar{K}_{R}$ goes through a peak of infinite height at the Brewster angle of the air-glass interface

$$
\begin{aligned}
\phi_{B 02}(\text { air-glass }) & =\tan ^{-1}\left(N_{\mathrm{glass}} / N_{\mathrm{arr}}\right) \\
& =\tan ^{-1} 1.5=56.31^{\circ} .
\end{aligned}
$$

This is true for all film materials and can be proved analytically as follows. At zero film thickness $\left(d_{1}=\right.$ $0, \beta=0$ ), Eq. (2) reduces to the identity

$$
R_{\nu}=r_{02 \nu}=\left(r_{01 \nu}+r_{12 \nu}\right) /\left(1+r_{01 \nu} r_{12 \nu}\right),(\nu=p, s),
$$

where $r_{02 \nu}$ are the Fresnel reflection coefficients for medium 0-medium 2 interface. For parallel-polarized light $(\nu=p)$ at the Brewster angle $\phi_{B 02}, r_{02 p}=0$, hence, from Eq. (44),

$$
r_{01 p}+r_{12 p}=0 \text { at } \phi=\phi_{B 02} \text {. }
$$

If we substitute $r_{01 p}+r_{12 p}=0$, the first term in the expression of $\bar{K}_{R}$ [Eq. (20)] becomes infinite.

(b) The angle $\bar{\theta}_{R}$ of the complex sensitivity factor $\bar{K}_{R}$ stays essentially constant near $\pm \pi / 2$ as the angle of incidence $\phi$ is increased from zero to 302 , experiences a discontinuous jump of $\pi$ at $\phi_{B 02}$, then stays essentially constant again near $\mp \pi / 2$ between $\phi_{B 02}$ and $90^{\circ}$. $\quad \bar{\theta}_{R}$ is exactly constant and equal to $-\pi / 2(0$ $\left.\leq \phi<\phi_{B 02}\right)$ or $+\pi / 2\left(\phi_{B 02}<\phi<90^{\circ}\right)$ only when the film is nonabsorbing, as in the case of the $\mathrm{MgF}_{2}$ film (Fig. 4). This can be seen as follows. When the three phases are transparent, all interface Fresnel reflection coefficients that appear in Eq. (20) become real. Thus the angle of $\bar{K}_{R}$ is that of $\gamma$ (except for an uncertainty of $\pm \pi$ ). From Eq. (8), we see that $\gamma$ is pure imaginary when $N_{1}$ is real, that is, when the film is nonabsorbing. Thus, $\bar{\theta}_{R}$ is either $\pi / 2$ or $-\pi / 2$. The extent to which $\bar{\theta}_{R}$ stays constant near $\pm \pi / 2$ depends on the amount of film absorption. This can be seen by comparing the cases of Si and Au films (Fig. 4).

(c) Although the functional dependence of $\left|\bar{K}_{R}\right|$ on $\phi$ is more or less the same for the three films considered $\left(\mathrm{MgF}_{2}, \mathrm{Si}\right.$, and $\left.\mathrm{Au}\right)$, values of $\left|\bar{K}_{R}\right|$ are quite different for the three films. By detuning the angle of incidence by $0.31^{\circ}$ below the Brewster angle $\phi_{B 02}$

\begin{tabular}{|c|c|c|c|c|c|c|c|}
\hline & \multicolumn{3}{|c|}{ Reflection $(R), \phi=56^{\circ}$} & & \multicolumn{3}{|c|}{ Transmission $(T), \phi=90^{\circ}$} \\
\hline & $\mathrm{MgF}_{2}$ & $\mathrm{Si}$ & $\mathrm{Au}$ & & $\mathrm{MgF}_{2}$ & $\mathrm{Si}$ & $\mathrm{Au}$ \\
\hline$\left|\bar{K}_{R}\right|(\mathrm{rad} / \AA)$ & 0.015 & 1.301 & 734.60 & $\left|\bar{K}_{T}\right|(\mathrm{rad} / \AA)$ & $1.6 \times 10^{-4}$ & $\begin{array}{c}1.368 \times \\
10^{-2}\end{array}$ & $\begin{array}{c}7.724 \times \\
10^{-3}\end{array}$ \\
\hline $\begin{array}{l}\bar{\theta}_{R}(\mathrm{deg}) \\
\bar{S}_{\Delta R}(\mathrm{deg} / \AA) \\
\bar{S}_{L P R}(\mathrm{deg} / \AA)\end{array}$ & $\begin{array}{l}90 \\
-0.872 \\
0\end{array}$ & $\begin{array}{r}89.030 \\
74.544 \\
0.011\end{array}$ & $\begin{array}{l}-73.065 \\
-4026.0 \\
0.104\end{array}$ & $\begin{array}{l}\bar{\theta}_{T}(\operatorname{deg}) \\
\bar{S}_{\Delta T}(\operatorname{deg} / \AA) \\
\bar{S}_{L T}(\operatorname{deg} / \AA)\end{array}$ & $\begin{array}{c}-90 \\
-0.009 \\
0\end{array}$ & $\begin{array}{c}89.0303 \\
0.785 \\
0.006\end{array}$ & $\begin{array}{c}-73.0728 \\
-0.423 \\
0.059\end{array}$ \\
\hline
\end{tabular}
(i.e., at $\phi=56^{\circ}$ ), we obtain the values of $\left|\bar{K}_{R}\right|$ given in Table I. From Table I, notice that the overall sensi-

Table I. External-Incidence Sensitivity Factors ${ }^{a}$

${ }^{a}$ The complex sensitivity factor $\bar{K}=|\bar{K}| \exp (j \bar{\theta})$ and the real psi and delta sensitivity factors $\bar{S}_{\psi}$ and $\bar{S}_{\Delta}$ determine the effect of the formation of a thin-film phase of thickness $\delta d_{1}$ at the interface between a transparent substrate and a transparent ambient on the ratio of reflection coefficients $\rho$ and the ellipsometric parameters $\psi$ and $\Delta$, according to $\delta \rho / \rho=\bar{K} \delta d_{1}, \delta \psi=\bar{S}_{\psi} \delta d_{1}, \delta \Delta=\bar{S}_{\Delta} \delta d_{1}$, respectively. 

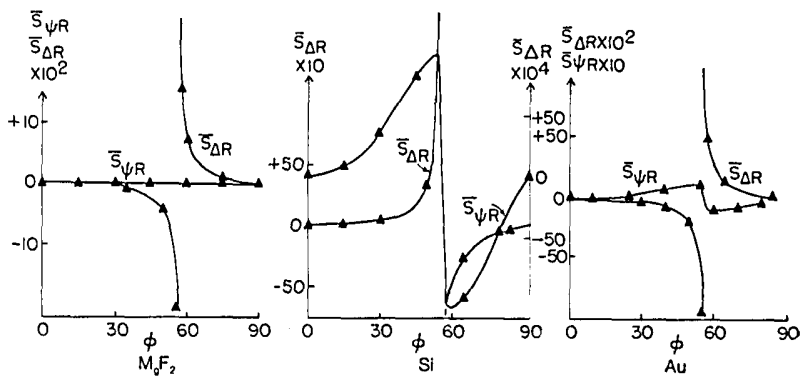

Fig. 5. Angle-of-incidence dependence of the psi and delta sensitivity functions $\bar{S}_{\psi R}$ and $\bar{S}_{\Delta R}$ for external reflection ellipsometry. In this and the subsequent figures $\bar{S}_{\psi}$ and $\bar{S}_{\Delta}$ are in units of degrees per angstrom. Left: air- $\mathrm{MgF}_{2}$-glass; middle: air-Si-glass; right: air-Au-glass.

tivity of the polarization ellipse of the externally reflected wave to the growth of thin films at the airglass interface is highest for metallic films ( $\mathrm{Au}$ ), followed by semiconducting films ( $\mathrm{Si})$, then by dielectric films $\left(\mathrm{MgF}_{2}\right)$. It is interesting to note that the sensitivity factor $\left|\bar{K}_{R}\right|$ covers a span of five decades (ratio of highest to lowest sensitivities is $\sim 10^{5}$ ) for the range of film materials assumed in the present study. The angles $\bar{\theta}_{R}$ that correspond to $\left|\bar{K}_{R}\right|$ are also given in Table I.

The psi and delta sensitivity factors $\bar{S}_{\phi R}$ and $\bar{S}_{\Delta R}$ are obtained from the complex sensitivity factor $\bar{K}_{R}$ by Eqs. (42). They are plotted in Fig. 5. The prominent characteristics of those curves are explained below.

(a) From Eq. (42b), we see that the delta sensitivity factor $\bar{S}_{\Delta R}$ is obtained by multiplying $\left|\bar{K}_{R}\right|$ by $\sin \bar{\theta}_{R}$ and a constant factor $180 / \pi$ that is required to convert $\bar{S}_{\Delta R}$ to units of degrees per angstrom. Because $\bar{\theta}_{R}$ stays essentially constant except for a change of $\pi$ at the Brewster angle $\phi_{B 02}, \bar{S}_{\triangle R}$ has the same behavior as $\pm\left|K_{R}\right|$ in the range $0<\phi \leq \phi_{B 02}$, and as $\mp\left|K_{R}\right|$ in the range $\phi_{B 02}<\phi \leq 90^{\circ}$. Therefore, the delta sensitivity factor $\bar{S}_{\Delta R}$ first increases (decreases) rapidly from zero to $+\infty(-\infty)$ as the angle of incidence $\phi$ is increased from zero (normal incidence) to the Brewster angle $\phi_{B 02}$, then it experiences a discontinuous jump to $-\infty(+\infty)$ at $\phi_{B 02}$, and finally it increases (decreases) from $-\infty(+\infty)$ to zero at $\phi$ continues to increase from $\phi_{B 02}$ to $90^{\circ}$ (grazing incidence). This behavior is the same for all films but again the values of $\bar{S}_{\Delta R}$ are different. At $\phi=56^{\circ}$ $\left(0.31^{\circ}\right.$ below the Brewster angle), the delta sensitivity factors are also given in Table I. Positive and negative $\bar{S}_{\Delta R}$ indicate that $\Delta$ increases or decreases, respectively, with increase of thickness of the thin-film phase, starting from the film-free substrate value $\bar{\Delta}$. The values of the delta sensitivity factor in Table I are very impressive. If the least detectable change of $\Delta$ is taken conservatively to be equal $0.05^{\circ}$, we reach the very interesting conclusion that films as thin as $0.1 \AA$ of $\mathrm{MgF}_{2}, 10^{-3} \AA$ of $\mathrm{Si}$, and $10^{-5} \AA$ of $\mathrm{Au}$ deposited on glass are easily measurable by external reflection ellipsometry near the Brewster angle. Notice that even higher sensitivities are attainable by detuning the angle of incidence by smaller amounts around the Brewster angle (less than $0.3^{\circ}$ ). We believe that the present possibility that ellipsometry is sensitive enough to detect a $10^{-5} \AA$ effective thickness of a film has not been reported in the literature. Of course, when we talk about subangstrom film thicknesses, we have to think of a sparse distribution of atoms or molecules (a submonolayer) sticking (adsorbed) to the substrate.

(b) From Eq. (42a), $\bar{S}_{\psi R}$ is obtained by multiplying $\left|\bar{K}_{R}\right|$ by three factors $F_{1}=\cos \bar{\theta}_{R}, F_{2}=1 / 2 \sin 2 \bar{\psi}_{R}$, and $F_{3}=180 / \pi$,

$$
\bar{S}_{\mho R}=F_{1} F_{2} F_{3}\left|\bar{K}_{R}\right| .
$$

The first of the three factors $F_{1}=\cos \bar{\theta}_{R}$ is identically zero for all transparent films, because $\bar{\theta}_{R}= \pm \pi / 2$. (See the discussion on $\vec{\theta}_{R}$ of Fig. 4.) Therefore,

$$
\bar{S}_{\psi R}=0 \text { at } 0 \leq \phi \leq 90^{\circ} \text {, }
$$

for all transparent films (see Fig. 4 for $\mathrm{MgF}_{2}$ ). Thus, to first order, the ellipsometric parameter $\psi_{R}$ is insensitive to the presence of a transparent thin-film phase between transparent ambient and substrate media. This is in agreement with the fact that the reflectances $\mathbf{R}_{\mathrm{p}}=\left|R_{p}\right|^{2}$ and $\mathbf{R}_{s}=\left|R_{s}\right|^{2}$ are insensitive, to first order, to the presence of transparent thin-film phases between transparent ambient and substrate media ${ }^{3}$ and that $\psi=\tan ^{-1}\left(\mathbf{R}_{p} / \mathbf{R}_{s}\right)^{1 / 2}$. When the film is absorbing, $\bar{\theta}_{R}$ is nearly a constant that is close, but not equal, to $\pm \pi / 2$ (Fig. 4). Therefore, $\cos \bar{\theta}_{R}$ is a small nonzero quantity that stays fairly unchanged with angle-of-incidence variations except that it switches sign at the Brewster angle. The second factor, $F_{2}=1 / 2 \sin 2 \bar{\psi}_{R}$, is a function of the angle of incidence $\phi$. It decreases from a maximum value of $1 / 2$ at $\phi=0\left(\bar{\psi}_{R}=\pi / 4\right)$ to zero at $\phi=\phi_{B 02}\left(\bar{\psi}_{R}=0\right)$, then increases again to $1 / 2$ at $\phi=90^{\circ}\left(\bar{\psi}_{R}=\pi / 4\right)$. Notice that the product $F_{2}\left|\bar{K}_{R}\right|$ becomes $0 \times \infty$ at the Brewster angle. It can be shown that this indeterminate product is actually zero, so that

$$
\bar{S}_{\psi R}=0 \text { at } \phi=\phi_{B 02}
$$

for any absorbing film. Because $\bar{S}_{\psi R}$ is zero also at $\phi$ $=0$ and $\phi=90^{\circ}$ and changes sign at $\phi=\phi_{B 02}$, it must show a minimum (maximum) in the interval $0<\phi<$ $\phi_{B 02}$ and a maximum (minimum) in the interval $\phi_{B 02}$ $<\phi<90^{\circ}$. Because of the behavior of $\left|\bar{K}_{R}\right|$ (Fig. 4), the angle of incidence at which $\bar{S}_{\psi R}$ is maximum or minimum should be close to the Brewster angle $\phi_{B 02}$. The cases of $\mathrm{MgF}_{2}, \mathrm{Si}$, and $\mathrm{Au}$ films are shown in Fig. 5. The psi sensitivity factors at $\phi=56^{\circ}$ are again given in Table I. These psi sensitivity factors are several (approximately four) orders of magnitude smaller than the corresponding delta sensitivity factors (Table I). We have already attributed this to the two terms $\cos \bar{\theta}_{R}$ and $1 / 2 \sin 2 \bar{\psi}$, each of which can become very small. In spite of this, very thin absorbing films (e.g., Si and $\mathrm{Au}$ ) of thicknesses of about $1 \AA$ are detectable in changes of the ellipsometric param- 


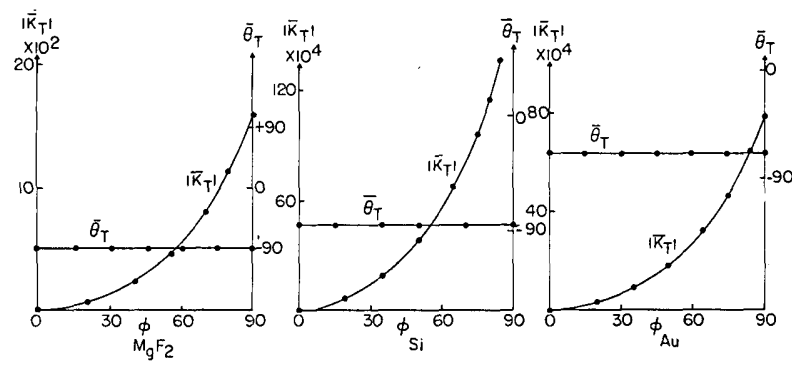

Fig. 6. Angle-of-incidence dependence of the magnitude $\left|\bar{K}_{T}\right|$ and angle $\bar{\theta}_{T}$ of the complex sensitivity function $\bar{K}_{T}$ for external-incidence transmission ellipsometry. Left: air- $\mathrm{MgF}_{2}$-glass; middle: air-Si-glass; right: air-Au-glass.

eter $\psi_{R}$ by use of instruments with precision that exceeds $0.01^{\circ}$.

\section{Transmission Ellipsometry ${ }^{22}$}

The magnitude $\left|\bar{K}_{T}\right|$ and angle $\bar{\theta}_{T}$ of the complex sensitivity factor $\bar{K}_{T}$ are plotted vs angle of incidence $\phi$ in Fig. 6 for the $\mathrm{MgF}_{2}, \mathrm{Si}$, and Au films. The most important features of these curves are the following.

(a) The magnitude $\left|\bar{K}_{T}\right|$ of the complex sensitivity factor $\bar{K}_{T}$ increases monotonically as a function of angle of incidence with no structure in the neighborhood of the Brewster angle of reflection $\phi_{B 02}$. This is true for all film materials. Maximum sensitivity occurs at grazing incidence $\left(\phi=90^{\circ}\right)$, with values given in Table I. From Table I it can be seen that the ellipse of polarization of the transmitted wave is one or two orders of magnitude less sensitive to the growth of transparent $\left(\mathrm{MgF}_{2}\right)$ films than to the growth of absorbing metallic $(\mathrm{Au})$ or semiconducting $(\mathrm{Si})$ films. Also, by comparing the values of $\left|\bar{K}_{T}\right|$ with those of $\left|\bar{K}_{R}\right|$ in Table I (the latter values pertain to the reflected wave near the Brewster angle), we see that transmission ellipsometry is about three to five orders of magnitude less sensitive to film growth than reflection ellipsometry for the same ambient-filmsubstrate systems.

(b) The angle $\bar{\theta}_{T}$ of the complex sensitivity factor $\bar{K}_{T}$ stays exactly constant at $-\pi / 2$ for the $\mathrm{MgF}_{2}$ film (Fig. 6) and for other transparent films. It is essentially constant near $-\pi / 2$ for the Si and Au films (Fig. 6) and for other absorbing films. In contrast with $\vec{\theta}_{R}$ in reflection, $\bar{\theta}_{T}$ does not experience any discontinuity at the Brewster angle or any other angle of incidence. That $\bar{\theta}_{T}=-\pi / 2$ for transparent films can be seen from Eqs. (8) and (30). When the three media are assumed to be transparent, all interface Fresnel reflection coefficients are real and the angle of $\bar{K}_{T}$ in Eq. (30) becomes equal to that of $\gamma$. From Eq. (8), in turn, $\gamma$ is pure imaginary when $N_{1}$ is real; therefore $\bar{\theta}_{T}=-\pi / 2$. The values of $\bar{\theta}_{T}$ at $\phi=90^{\circ}$ are listed in Table I.

The psi and delta sensitivity factors $\bar{S}_{\psi T}$ and $\bar{S}_{\Delta T}$ for transmission ellipsometry are obtained from $\bar{K}_{T}$ by Eqs. (42). They are plotted in Fig. 7 for the
$\mathrm{MgF}_{2}, \mathrm{Si}$, and $\mathrm{Au}$ films. The salient characteristics of these curves are as follows.

(a) Because $\bar{S}_{\Delta T} \alpha\left|\bar{K}_{T}\right| \sin \bar{\theta}_{T}$, Eq. (42b), and $\bar{\theta}_{T}$ are essentially (exactly for the $\mathrm{MgF}_{2}$ film) constant, $\bar{S}_{\Delta T}$ has the same form as $\left|\bar{K}_{T}\right|$ when plotted as a function of the angle of incidence $\phi .\left|\bar{S}_{\Delta T}\right|$ increases monotonically with $\phi$ to a maximum value at $\phi=90^{\circ}$ (grazing incidence), given in Table I. The transmission sensitivities that appear in Table I are very significant, although they are three to five orders of magnitude smaller than the corresponding reflection sensitivities that appear in the same table. They show that the growth of very thin films (down to $0.1 \AA$ for $\mathrm{Si}$ and $\mathrm{Au}$ ) is quite measurable by monitoring the ellipsometric parameter $\Delta_{T}$ of the transmitted wave.

(b) Because $\bar{S}_{\psi T} \alpha\left(\sin 2 \bar{\psi}_{T}\right) \cos \bar{\theta}_{T}$, Eq. (42a), we have

$$
\bar{S}_{\psi T}=0 \text { at } 0 \leq \phi \leq 90^{\circ},
$$

when $\bar{\theta}_{T}= \pm 90$, i.e., for all transparent films. Therefore, we conclude that the growth of very thin films in an all transparent three-phase systems is not observable, to first order, in changes of the ellipsometric parameter $\psi_{T} . \bar{\psi}_{T}$, the bare substrate value of $\psi_{T}$, decreases monotonically but gently as a function of the angle of incidence between $\phi=0$ and $\phi=90^{\circ}$. The increase of $\left|\bar{K}_{T}\right|$ with $\phi$, however, is more rapid, with the result that $\left|\bar{S}_{\psi T}\right|$ increases monotonically with angle of incidence. The maximum values of $\bar{S}_{\psi T}$ occur at grazing incidence and are given in Table I. These values are not significantly different from those obtained in reflection ellipsometry near the Brewster angle. The transmission psi sensitivity factors are large enough to make 1 - $\AA$ absorbing films detectable by transmission ellipsometry, using an instrument with $\sim 0.005^{\circ}$ precision.

\section{B. Internal Incidence}

It is important to examine how the ellipsometric ratios $\rho_{R}=\tan \psi_{R} \exp \left(j \Delta_{R}\right)$ and $\rho_{T}=\tan \psi_{T} \exp \left(j \Delta_{T}\right)$, as well as the complex sensitivity functions $\bar{K}_{R}$ and $\bar{K}_{T}$ that describe light reflection $(R)$ and transmission $(T)$, are affected when the direction of wave propagation is reversed, so that incidence takes place

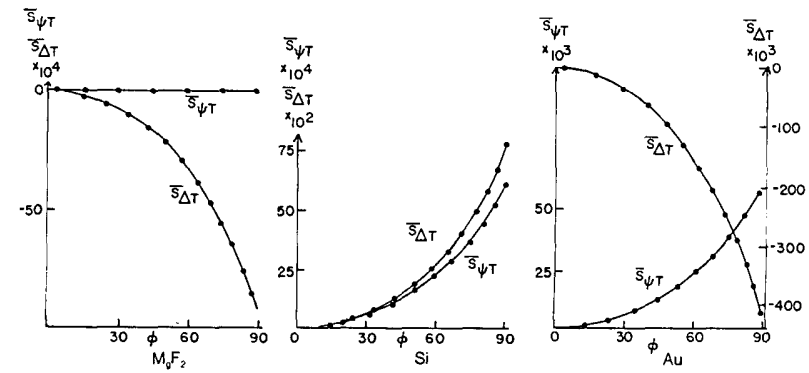

Fig. 7. Angle-of-incidence dependence of the psi and delta sensitivity functions $\bar{S}_{\psi T}$ and $\bar{S}_{\Delta T}$ for external-incidence transmission ellipsometry. Left: air- $\mathrm{MgF}_{2}$-glass; middle: air-Si-glass; right: air-Au-glass. 


\begin{tabular}{|c|c|c|c|c|c|}
\hline \multirow[b]{2}{*}{ Quantity } & \multicolumn{2}{|c|}{ Reflection } & \multicolumn{2}{|c|}{ Transmission } & \multirow[b]{2}{*}{ Remarks } \\
\hline & External & Internal & External & Internal & \\
\hline $\begin{array}{l}\text { Complex amplitude-reflection } \\
\text { or transmission coefficient } \\
\text { for } p \text { polarization }\end{array}$ & $R_{p}$ & $-R_{p}$ & $T_{p}$ & $\alpha_{p} T_{p}$ & $\begin{aligned} & \alpha_{\nu}= {\left[\left(1-r_{01 \nu}^{2}\right)\left(1-r_{12 \nu}^{2}\right)\right] / } \\
& t_{01 \nu} t_{12 \nu}\end{aligned}$ \\
\hline $\begin{array}{l}\text { Complex amplitude-reflection } \\
\text { or transmission coefficient } \\
\text { for } s \text { polarization }\end{array}$ & $R_{s}$ & $-R_{s}$ & $T_{s}$ & $\alpha_{s} T_{s}$ & $\nu=p, s$ \\
\hline Ellipsometric ratios $\rho$ & $\rho_{R}$ & $\rho_{R}$ & $\rho_{T}$ & $\left(\alpha_{p} / \alpha_{s}\right)_{\rho T}$ & $\begin{array}{l}\rho_{R}=R_{p} / R_{s} \\
\rho_{T}=T_{p} / T_{s}\end{array}$ \\
\hline Complex sensitivity functions & $\bar{K}_{R}$ & $\bar{K}_{R}$ & $\bar{K}_{T}$ & $\bar{K}_{T}$ & $\begin{array}{l}\delta d_{1} \text { is the thickness of the thin } \\
\text { film }\end{array}$ \\
\hline
\end{tabular}

from the glass side instead of the air side of the glassfilm-air interface. It can be shown that the interface Fresnel reflection $r$ and transmission $t$ coefficients transform upon a reversal of the direction of propagation to $r^{\prime}$ and $t^{\prime}$ such that

$$
\begin{gathered}
r^{\prime}=-r, \\
t^{\prime}=\left(1-r^{2}\right) / t .
\end{gathered}
$$

The law of transformation of the three-phase reflection $\left(R_{p^{\prime}}, R_{s^{\prime}}\right)$ and transmission $\left(T_{p^{\prime}}, T_{s^{\prime}}\right)$ coefficients and that of the ellipsometric ratios $\rho_{R^{\prime}}, \rho_{T} T^{\prime}$ and the complex sensitivity functions $\bar{K}_{R^{\prime}}, \bar{K}_{T^{\prime}}$ can be obtained by substituting Eqs. (50) into Eqs. (2) and (22), Eqs. (1) and (21), and Eqs. (20) and (28), respectively. The results are summarized in Table II. From this table, we see that when the direction of propagation is reversed, the ellipsometric ratio of reflection coefficients $\rho_{R^{\prime}}$ and the complex reflection and transmission sensitivity factors $\bar{K}_{R^{\prime}}$ and $\bar{K}_{T^{\prime}}$ stay the same, whereas the ellipsometric ratio of transmission coefficients $\rho_{T^{\prime}}$ is changed. Alternatively, we can say that for each angle of external incidence $\phi_{0}$, there is a corresponding angle of internal incidence $\phi_{2}$ that leads to the same ratio of reflection coefficients $\rho_{R}$ and the same ellipsometric sensitivities $\bar{K}_{R}$ and $\bar{K}_{T}$ in reflection and transmission; these two angles are related to each other by Snell's law

$$
N_{0} \sin \phi_{0}=N_{2} \sin \phi_{2}, N_{2}>N_{1} \text {. }
$$

The opposite of the above statement is not true. For each angle of internal incidence $\phi_{2}$ there may or may not be a corresponding angle of external incidence $\phi_{0}$ such that $\rho_{R}, \bar{K}_{R}$, and $\bar{K}_{T^{\prime}}$ are the same. The reason is that total reflection occurs when the angle of internal incidence $\phi_{2}$ exceeds the critical angle

$$
\begin{aligned}
\phi_{C 20} & =\sin ^{-1}\left(N_{0} / N_{2}\right), \\
& =\sin ^{-1}(0.6667)=41.82^{\circ},
\end{aligned}
$$

for the glass-air interface. At the critical angle, the transmitted wave in air grazes parallel to the glassair interface. For all angles $\phi_{2}>\phi_{C 20}$ the transmitted wave becomes evanescent, hence inaccessible for ellipsometry. This is a situation that could not be reproduced at any angle of external incidence.
In the light of the above discussion, we need only emphasize the main differences between internal and external incidence and reflection and transmission ellipsometry.

\section{Reflection Ellipsometry ${ }^{23}$}

Figure 8 shows the sensitivity functions $\left|\bar{K}_{R^{\prime}}\right|, \theta_{R^{\prime}}$, and $\left(\bar{S}_{\psi_{R^{\prime}}}, \bar{S}_{\Delta^{\prime}}\right)$, respectively, for the glass-Si-air system. Other film materials $\left(\mathrm{MgF}_{2}\right.$ and $\left.\mathrm{Au}\right)$ lead to the same behavior, only the magnitudes are different. For the $\mathrm{MgF}_{2}$ film (and any other transparent film as well), $\bar{S}_{\psi_{R^{\prime}}}=0$, i.e., transparent film formation does not produce changes in $\psi_{R}$, in internal reflection to first order. A similar conclusion was encountered earlier for external reflection. Notice that $\left|\bar{K}_{R^{\prime}}\right|$ and $\left|\bar{S}_{\Delta R^{\prime}}\right|$ become infinitely large at the Brewster angle of internal incidence

$$
\begin{aligned}
\phi_{B 20} & =\tan ^{-1}\left(N_{0} / N_{2}\right) \\
& =90^{\circ}-\tan ^{-1}\left(N_{2} / N_{0}\right) \\
& =90^{\circ}-\phi_{B 02} \\
& =33.69^{\circ},
\end{aligned}
$$

and that $\bar{\theta}_{R^{\prime}}$ experiences a discontinuity at that angle. Very high sensitivities are attainable near $\phi_{B 20}$ in internal incidence as they were realizable near $\phi_{B 02}$ in external incidence.

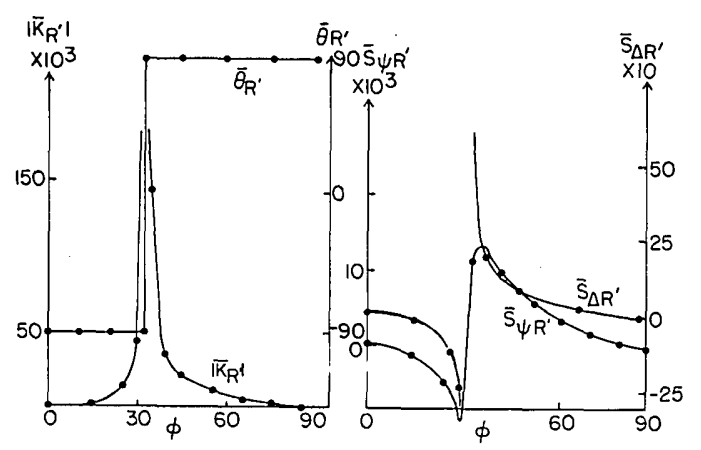

Fig. 8. Angle-of-incidence dependence of the magnitude $\left.\mid \bar{K}_{R}\right\}$ and angle $\bar{\theta}_{R^{\prime}}$ of the complex sensitivity function $\bar{K}_{R^{\prime}}$ and of the psi and delta sensitivity functions $\bar{S}_{\psi R^{\prime}}$ and $\bar{S}_{\Delta R^{\prime}}$ for internal reflection ellipsometry. Glass-Si-air. 


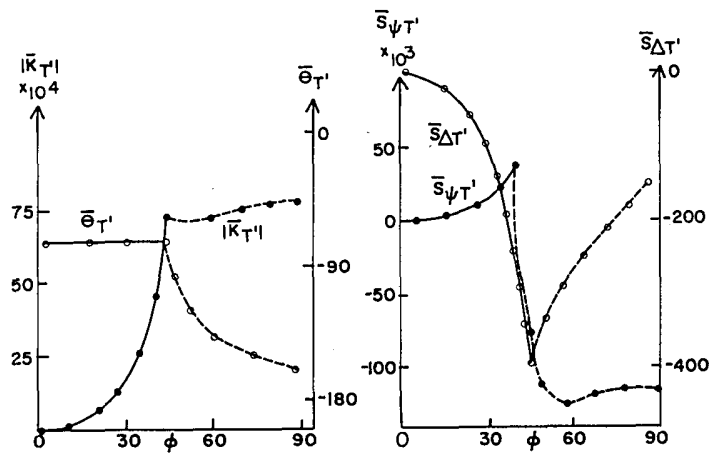

Fig. 9. Angle-of-incidence dependence of the magnitude $\left|\bar{K}_{T}\right|$ and angle $\bar{\theta}_{T^{\prime}}$ of the complex sensitivity function $\bar{K}_{T^{\prime}}$ and of the psi and delta sensitivity functions $\bar{S}_{\psi T^{\prime}}$ and $\bar{S}_{\Delta T^{\prime}}$ for internal-incidence transmission ellipsometry. Glass-Au-air.

One interesting observation that can be made from Fig. 8 (as well as from the corresponding figures for the $\mathrm{MgF}_{2}$ and $\mathrm{Au}$ films, which are not shown here for sake of brevity) is that no structure exists in any of the three ellipsometric sensitivity functions $\left.\mid \bar{K}_{R}\right\}, \bar{S}_{\psi R^{\prime}}$, and $\bar{S}_{\Delta R^{\prime}}$ at or near the angle of total internal reflection. The only angle of importance in internal-reflection ellipsometry is the Brewster angle $\phi_{B 20}$, Eq. (53).

\section{Transmission Ellipsometry}

The sensitivity functions $\bar{K}_{T^{\prime}}, \bar{S}_{\psi T^{\prime}}$, and $\bar{S}_{\Delta T^{\prime}}$ in internal-transmission ellipsometry for a glass-Au-air system are shown in Fig. 9. It is evident that the behavior of such functions in the internal $0 \leq \phi \leq \phi_{C 20}$ is a compressed version of the behavior of the same quantities in external transmission over the extended interval $0 \leq \phi \leq 90^{\circ}$. These features also apply to other films $\left(\mathrm{MgF}_{2}, \mathrm{Si}\right)$. Maximum sensitivities in internal transmission occur at the critical angle $\left(\phi_{C 20}=\right.$ $\left.41.816^{\circ}\right)$. They are equal to the external-transmission sensitivities at grazing incidence. These conclusions are corollaries of the relationship that we proved earlier connecting internal and external incidence. When the angle of incidence is greater than the critical angle, the transmitted wave is evanescent. In Fig. 9, portions of the curves over the interval $\phi_{C 20}$ $<\phi \leq 90^{\circ}$ are shown as dashed lines. They may be considered as analytical continuation of the sensitivity functions in this evanescent-wave domain.

\section{Validity of the Linear Approximations}

Because the sensitivity considerations that we have discussed so far are based on linear approximations of the exact equations of reflection [Eqs. (1) and (2)] and transmission [Eq. (23)] ellipsometry, it is important to examine the range of thickness values over which such approximations are of acceptable accuracy. We have computed curves for $\psi$ and $\Delta$ as functions of film thickness in the range $0-300 \AA$ based on the exact equations of ellipsometry. These curves are compared with straight lines drawn through the point $\bar{\psi}, \bar{\Delta}$, which characterizes the film-free ambient- substrate interface, with slopes given by the psi and sensitivity factors $\bar{S}_{\psi}$ and $\bar{S}_{\Delta}$, respectively.

The case of external reflection near the Brewster angle for an air- $\mathrm{MgF}_{2}$-glass system is shown in Fig. 10. Because $\bar{S}_{\psi R}=0$ for transparent films [Eq. (47)], $\psi_{R}$ increases parabolically with thickness. This restricts the linear range for $\psi$ to subangstrom thicknesses near the minimum of the parabola. The linearity of $\Delta_{R}$ holds for thicknesses up to $30 \AA$. The case of external transmission at $\phi=75^{\circ}$, which represents a compromise between high sensitivity near grazing incidence and experimental convenience, for the same system, is also shown in Fig. 10. Because $\bar{S}_{\psi T}=0$ for transparent films, $\psi_{T}$ is again a parabolic function of film thickness. The linearity ranges for $\psi_{T}$ and $\Delta_{T}$ are less than $5 \AA$ in this case.

Figure 11 (left) shows the results for external reflection at $\phi=75^{\circ}$ for the air-Si-glass system. The linear ranges for $\psi$ and $\Delta$ are about $1 \AA$ and $10 \AA$, respectively. Transmission ellipsometry on the same system at the same angle of incidence, Fig. 11 (right), indicates that the linear approximations of $\psi_{T}$ and $\Delta_{T}$ are valid over thickness ranges of $2 \AA$ and $20 \AA$, respectively.

Results for external reflection and transmission from the air-Au-glass system at $\phi=75^{\circ}$ are shown in

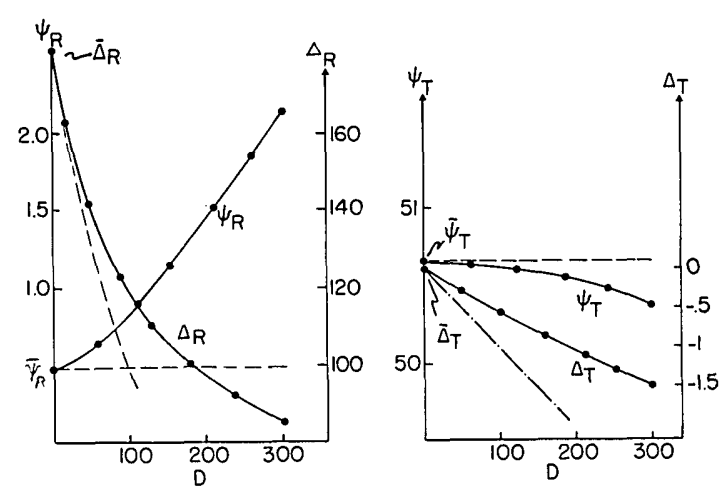

Fig. 10. Dependence of the ellipsometric angles psi $(\psi)$ and delta $(\Delta)$ on the film thickness $D\left(=d_{1}\right)$ for the air- $\mathrm{MgF}_{2}$-glass system. In this and the subsequent figures $\psi$ and $\Delta$ are in degrees. $D$ is in angstroms. Left: external-reflection ellipsometry; right: external-incidence transmission ellipsometry.

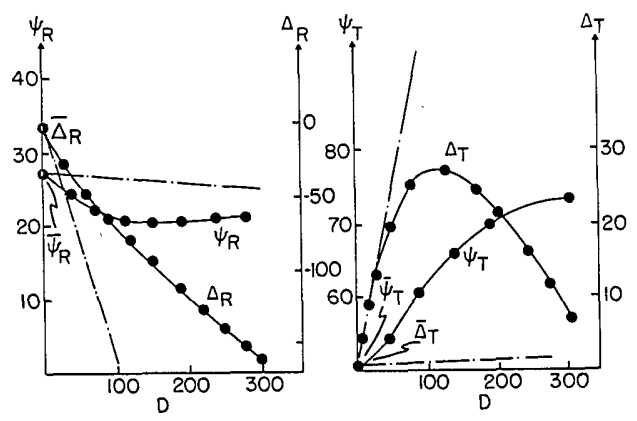

Fig. 11. Thickness dependence of psi and delta for the air-Siglass system. Left: external-reflection ellipsometry; right: external-incidence transmission ellipsometry. 

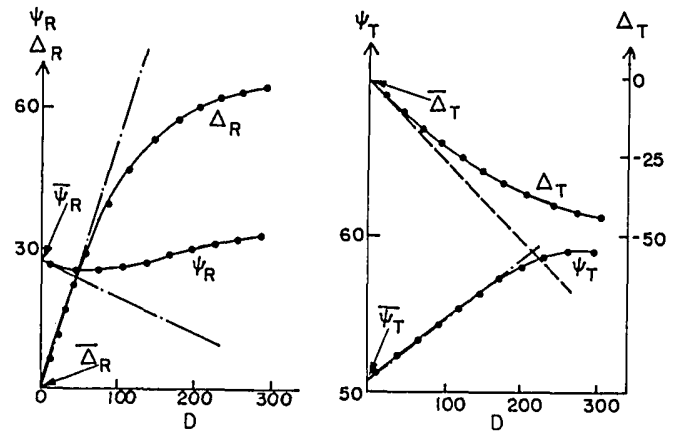

Fig. 12. Thickness dependence of psi and delta for the air-Auglass system. Left: external-reflection ellipsometry; right: external-incidence transmission ellipsometry.

Fig. 12. Notice that $\psi_{T}$ is obtained from the linear approximation for thicknesses up to about $140 \AA$.

Examples of total internal reflection ellipsometry are shown in Fig. 13 for the glass-Si-air (right) and glass-Au-air (left) systems, respectively, at $\phi=75^{\circ}$. The maximum thicknesses for linearity of $\psi$ and $\Delta$ are about $10-20 \AA$. In Fig. 13 (left), it is interesting to observe the considerable structure in the curves in the thickness range $0-300 \AA$ for $\mathrm{Au}$. This is not unexpected, because of the strong influence that the film exerts on the evanescent wave, which has a spatial extent of the same order of magnitude as the film thickness.

The above examples show that the linear approximations in external and internal reflection and transmission ellipsometry are generally applicable for the growth of very thin films $(\sim 10 \AA)$ and are sometimes further limited to submonolayer coverage $(\sim 1 \AA)$. This is a range of thickness of considerable interest for the application of ellipsometry to the detection of extremely small amounts of adsorbed atomic or molecular species at interfaces. An obvious conclusion to reach from the above examples is that the range of validity of the linear approximation is quite dependent on the particular system under consideration.

One important consideration in the experimental application of the above results is the substrate roughness. However, it has been shown experimentally that ellipsometry gives valid results for sparse coverage on smooth surfaces. ${ }^{24,25}$ Moderate surface roughness, such as that resulting from mildly etched surfaces, may be tolerated if zone averaging is used. ${ }^{26}$

\section{v. Conclusions}

The important conclusions of this paper are summarized below.

1. A scheme of combined reflection and transmission ellipsometry on light-transmitting ambientfilm-substrate systems is proposed. Sample design and instrument operation that are required for this technique are investigated in Sec. II.

2. A comparative study of sensitivity of reflection and transmission ellipsometry is carried out based on a unified analysis of the linear approximation of the exact equations in Sec. III. These linear approximations are general in that an arbitrary initial film thickness is assumed. They are also simple because a complex sensitivity function $\bar{K}$ is used. The real and imaginary projections of this function give the psi $(\psi)$ and delta $(\Delta)$ sensitivity factors, respectively. By leaving $\bar{K}$ expressed in terms of the interface Fresnel reflection coefficients instead of the optical constants of the three media, unnecessary complexity and erroneous results are avoided.

3. External reflection ellipsometry near the Brewster angle of a transparent ambient-substrate system is extremely sensitive to the presence of very thin films at the interface. By detuning the angle of incidence by $0.3^{\circ}$ below the Brewster angle of an airglass interface, films as thin as $0.1 \AA$ of $\mathrm{MgF}_{2}, 10^{-3} \AA$ of $\mathrm{Si}$, and $10^{-5} \AA$ of $\mathrm{Au}$ deposited on glass are readily detectable by an ellipsometer with $0.05^{\circ}$ precision (Sec. IV). These subangstrom films would represent sparse distributions of atoms or molecules at the interface.

4. In internal and external reflection and transmission ellipsometry on an all-transparent threephase system, the formation of a nonabsorbing thin film is not detectable, to first order, as a change in the ellipsometric parameter $\psi$. In this case, the psi sensitivity functions $\bar{S}_{\psi R}$ and $\bar{S}_{\psi T}$ are identically zero at all angles of incidence.

5. The detection sensitivity of thin films by transmission ellipsometry increases monotonically with angle of incidence to reach a maximum value at external grazing incidence and at the critical angle of total internal reflection. The absolute delta sensitivity values $\left|\bar{S}_{\Delta T}\right|$ of transmission ellipsometry are large enough to make absorbing films that are as thin as $0.1 \AA$ readily measurable. Film thickness must exceed $\sim 1 \AA$ for detectability as a change of $\psi$.

6. For each angle of external incidence, there is a corresponding angle of internal incidence that leads to the same ratio of reflection coefficients $\rho_{R}$ and the same ellipsometric sensitivities $\bar{K}_{R}$ and $\bar{K}_{T}$. These angles are interrelated by Snell's law. The ratio of transmission coefficients $\rho_{T}$ is changed upon reversal of the direction of light propagation.

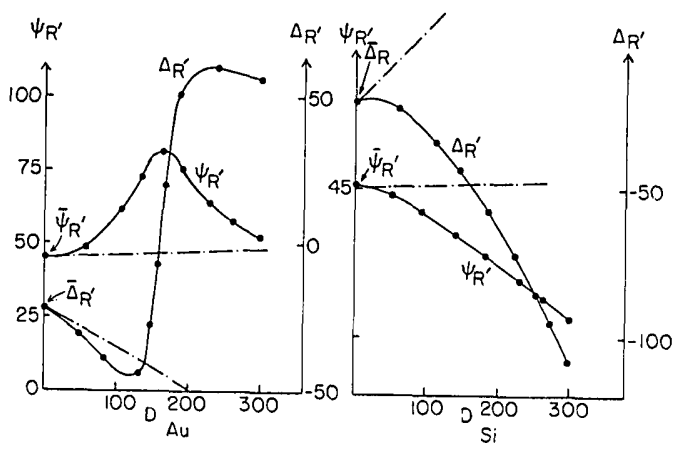

Fig. 13. Thickness dependence of the psi and delta for the glass$\mathrm{Au}$-air system (left) and the glass-Si-air system (right) in totalinternal-reflection ellipsometry. 
7. The ranges of validity of the linear approximation in reflection and transmission ellipsometry are comparable. Such ranges may be as small as $1 \AA$ and as large as $150 \AA$. Usually, the linear range for $\psi$ is more restricted than that for $\Delta$. The case of total internal reflection ellipsometry leads to very strong nonlinear behavior of $\psi_{R}$ and $\Delta_{R}$ as functions of film thicknesses, in the range below 0.05 of the wavelength of light.

This research was supported by the National Science Foundation. R. M. A. Azzam is also with the Hematology Division, Department of Internal Medicine, University of Nebraska Medical Center, Omaha, Nebraska 68105.

\section{References}

1. E. Passaglia, R. R. Stromberg, and J. Kruger, Eds., Ellipsometry in the Measurement of Surfaces and Thin Films, Natl. Bur. Std. Misc. Pub. 256 (U.S. Govt. Printing Office, Washington, 1964); N. M. Bashara, A. B. Buckman, and A. C. Hall, Eds., Proceedings of the Symposium on Recent Developments in Ellipsometry (North-Holland, Amsterdam, 1969) [Surface Sci. 16, 1969].

2. J. D. E. McIntyre and W. Hansen, in Advances in Electrochemistry and Electrochemical Engineering, R. H. Muller, Ed. (Wiley-Interscience, New York, 1973), Vol. 9, Chaps. 1 and 2.

3. J. D. E. McIntyre and D. E. Aspnes, Surface Sci. 24, 417 (1971).

4. It may be necessary to choose a measuring wavelength such that these requirements are met over the thickness range of interest.

5. B. D. Cahan and R. F. Spanier, Surface Sci. 16, 166 (1969).

6. D. E. Aspnes, Opt. Commun. 8, 222 (1973).

7. P. S. Hauge and F. H. Dill, IBM J Res. Devel. 17, 472 (1973).
8. S. N. Jasperson, D. K. Burge, and R. C. O'Handley, Surface Sci. 37, 548 (1973).

9. J. L. Ord, Surface Sci. 16, 155 (1969).

10. D. E. Confer, R. M. A. Azzam, and N. M. Bashara, to be published.

11. In differential reflection spectroscopy, we measure the fractional change of reflectance $\delta \mathbf{R}_{\nu} / \mathbf{R}_{\nu}$, where $\mathbf{R}_{\nu}=R_{\nu} R_{\nu}{ }^{*}$. We can write $\delta \mathbf{R}_{v} / \mathbf{R}_{\nu}=\left(\delta R_{\nu} / R_{\nu}\right)+\left(\delta R_{\nu}{ }^{*} / R_{\nu}{ }^{*}\right)=2 \operatorname{Re}\left(\delta R_{\nu} / R_{\nu}\right)=$ $\left[2 \operatorname{Re}\left(Q_{\nu}\right)\right]\left(\delta d_{1} / d_{1}\right)$, where $Q_{\nu}$ is given by Eq. (14). This result is in agreement with one obtained by McIntyre and Aspnes (Ref. 3).

12. The zero-thickness psi and delta sensitivity factors for reflection ellipsometry were introduced by R. C. Smith and M. Hacskaylo (the first of Refs. 1, p. 83), who used the restricted Drude linear approximation.

13. L. Tronstad, Trans. Faraday Soc. 31, 1151 (1935).

14. C. E. Lebernight and B. Lustman, J. Opt. Soc. Am. 29, 59 (1939).

15. R. J. Archer, J. Electrochem. Soc. 104, 619 (1957).

16. D. K. Burge and H. E. Bennett, J. Opt. Soc. Am. 54, 1428 (1964).

17. R. J. Archer, Manual on Ellipsometry (Gaertner Sci. Corp., Chicago, 1968).

18. D. W. Peterson and N. M. Bashara, J. Opt. Soc. Am. 55, 845 (1965).

19. A. N. Saxena, J. Opt. Soc. Am. 55, 1061 (1965).

20. E. C. Rowe and I. Shewchun, J. Opt. Soc. Am. 59, 1385 (1969).

21. Burge and Bennett, Ref. 16, have pointed out several errors in the linear approximations used by earlier workers.

22. Transmission ellipsometry on glass slides that are coated on both sides by anisotropic Langmuir-Blodgett layers were studied by D. den Engelsen, J. Phys. Chem. 76, 3390 (1972).

23. Internal reflection ellipsometry was used by E. C. Chan and J. P. Marton, J. Appl. Phy. 43, 4027 (1972), to study metal deposits on glass slides. See also M. J. Dignam, B. Rao, M. Moskovits, and R. B. Stobie, Can. J. Chem. 49, 1115 (1971).

24. Tennyson Smith, J. Opt. Soc. Am. 58, 1069 (1968).

25. G. A. Bootsma and F. Meyer, Surface Sci. 14, 52 (1969).

26. R. M. A. Azzam and N. M. Bashara, J. Opt. Soc. Am. 61, 1236 (1971).

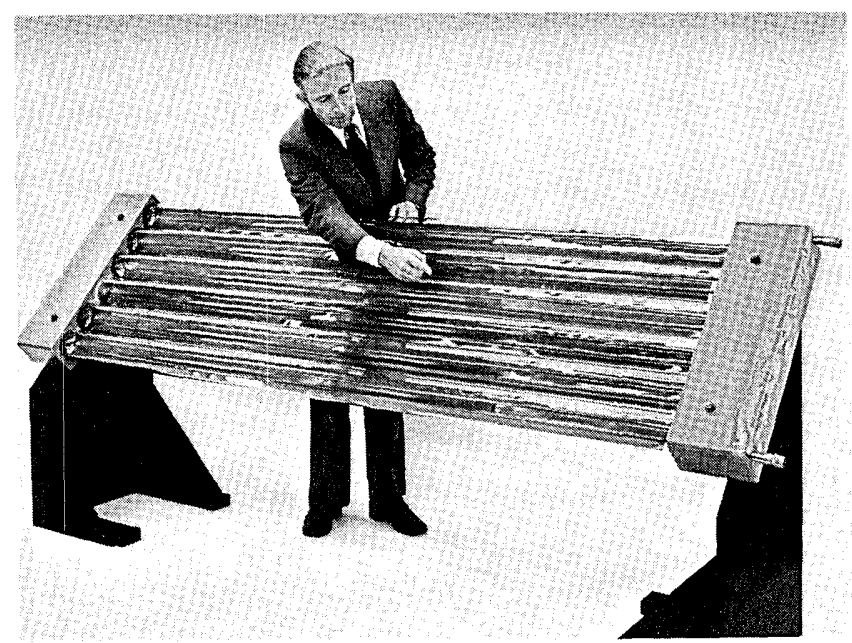

The new high-performance tubular solar collector developed by Corning Glass works incorporates a selectively coated flat absorber plate housed within a highly evacuated glass tube. The individual collector tubes are mounted in modules of from six (as shown) to ten tubes. Corning said it has begun manufacture of the collector in limited quantities for experimental and demonstration purposes. The firm is now cooperating in the planning of both large and small solar projects which will reauire delivery of collectors beginning in mid-1975. 